\title{
Generalized Integral inequalities for Hermite-Hadamard type via $s$-convexity on fractal sets
}

\author{
Ohud Almutairi ${ }^{1}$ and Adem Kılıçman ${ }^{2}$ \\ Department of Mathematics, University of Hafr Al-Batin \\ Hafr Al-Batin 31991, Sudia Arabia \\ Universiti Putra Malaysia, Serdang 43400 UPM, Selangor, Malaysia \\ ${ }^{1}$ AhoudbAlmutairi@gmail.com and ${ }^{2}$ akilic@upm.edu.my
}

November 14, 2019

\begin{abstract}
In this article, the new HermiteHadamard type inequalities are studied via generalized $s$-convexity on fractal sets. These inequalities derived on fractal sets are shown to be the generalized $s$-convexity on fractal sets. We proved that the absolute values of the first and second derivatives for the new inequalities are the generalization of $s$-convexity on fractal sets.
\end{abstract}

Keywords: $s$-convex function; Hermite-Hadamard inequalities; Riemann-Liouville fractional inte-grals; fractal space

\section{Introduction}

The convexity is considered among the important property in mathematical analysis. The applications of convex functions can be found in many fields of studies including economy, engineering and optimization (see for example [1,4]). A well-known result which was identified as Hermite-Hadamard inequalities is the reformulation through the convexity. These inequalities, widely reported in the literature, can be defined as follows:

Theorem 1. Suppose that $\psi:[u, v] \subset \mathbb{R} \rightarrow \mathbb{R}$ is a convex function on $[u, v]$ with $u<v$, then

$$
\left(\frac{u+v}{2}\right) \leq \frac{1}{v-u} \int_{u}^{v} \psi(x) d x \leq \frac{\psi(u)+\psi(v)}{2} .
$$

These two inequalities, which are the refinement to the convexity, can be held in reverse order as concave. Following this, many refinements of convex functions using Hermite-Hadamard inequalities have been continuously studied [3, 5, 7]. Given the variation of Hermite-Hadamard inequalities, Dragomir and Fitzpatrick [6] established a new generalizations of $s$-convex functions in the second sense. 
Theorem 2. Suppose that $\psi: \mathbb{R}_{+} \rightarrow \mathbb{R}_{+}$is a s-convex function in the second sense, where $0<s \leq 1, u, v \in \mathbb{R}_{+}$and $u<v$. If $\psi \in L^{1}([u, v])$, then

$$
2^{s-1} \psi\left(\frac{u+v}{2}\right) \leq \frac{1}{v-u} \int_{u}^{v} \psi(x) d x \leq \frac{\psi(u)+\psi(v)}{s+1} .
$$

Even though the Hermite-Hadamard inequalities are established for classical integrals, the inequalities can also hold for fractional calculus. Other important generalizations include the work of Sarkaya et al. [8], who proved the Hermite-Hadamard inequalities through fractional integrals as follows:

Theorem 3. Suppose that $\psi:[u, v] \rightarrow \mathbb{R}$ is a non-negative function with $0 \leq u<v$ and $\psi \in$ $L_{1}[u, v]$. If $\psi$ is convex function on $[u, v]$, we have:

$$
\psi\left(\frac{u+v}{2}\right) \leq \frac{\Gamma(\alpha+1)}{2(v-u)^{\alpha}}\left[J_{u^{+}}^{\alpha}(v)+J_{v}^{\alpha}(u)\right] \leq \frac{\psi(u)+\psi(v)}{2},
$$

where $\alpha \geq 0$.

The $s$-convexity mentioned in Hudzik and Maligranda [12] was also given as the generalization on fractal sets.

Definition 1. [9] A function $\psi: \mathbb{R}_{+} \rightarrow \mathbb{R}^{\alpha}$ is called generalized $s$-convex in the second sense if

$$
\psi\left(\gamma_{1} u+\gamma_{2} v\right) \leq \gamma_{1}^{\alpha s} \psi(u)+\gamma_{1}^{\alpha s} \psi(v)
$$

holds for all $u, v \in \mathbb{R}_{+}, \gamma_{1}, \gamma_{2} \geq 0$, with $\gamma_{1}+\gamma_{2}=1$ and for some fixed $s \in(0,1]$. The symbol $G K_{s}^{2}$ denotes the class of this function .

The Riemann-Liouvile fractional integral is introduced here due to its importance.

Definition 2. [10] Suppose that $\psi \in L_{1}[u, v]$. The Riemann-Liouvile integrals $J_{u+}^{\alpha} \psi$ and $J_{v-}^{\alpha} \psi$ of order $\alpha \in \mathbb{R}_{+}$are defined by

$$
J_{u+}^{\alpha} \psi(x)=\frac{1}{\Gamma(\alpha)} \int_{u}^{x}(x-\gamma)^{\alpha-1} f(\gamma) d \gamma, \quad x>u,
$$

and

$$
J_{v-}^{\alpha} \psi(x)=\frac{1}{\Gamma(\alpha)} \int_{x}^{v}(\gamma-x)^{\alpha-1} f(\gamma) d \gamma, \quad x<v
$$

respectively.

The following lemma for differentiable mapping is given by Sarikaya et al. [8].

Lemma 1. Let $\psi:[u, v] \rightarrow \mathbb{R}$ be a differentiable mapping on $(u, v)$ with $u<v$. If $\psi^{\prime} \in L[u, v]$, then we have:

$$
\frac{\psi(u)+\psi(v)}{2}-\frac{\Gamma(\alpha+1)}{2(v-u)^{\alpha}}\left[J_{u+}^{\alpha} \psi(v)+J_{v-}^{\alpha} \psi(u)\right]=\frac{v-u}{2} \int_{0}^{1}\left[(1-\gamma)^{\alpha}-\gamma^{\alpha}\right] \psi^{\prime}(\gamma u+(1-\gamma) v) d \gamma
$$


Wang et al. [11] extended Lemma 1 to include two casses, one of wich involves the second derivative of Riemann-Liouvile fractional integrals.

Lemma 2. Let $\psi:[u, v] \rightarrow \mathbb{R}$ be a twice-differentiable mapping on $(u, v)$ with $u<v$. If $\psi^{\prime \prime} \in$ $L^{1}[u, v]$, then

$$
\begin{aligned}
& \frac{\psi(u)+\psi(v)}{2}-\frac{\Gamma(\alpha+1)}{2(v-u)^{\alpha}}\left[J_{u+}^{\alpha} \psi(v)+J_{v-}^{\alpha} \psi(u)\right] \\
= & \frac{(v-u)^{2}}{2} \int_{0}^{1} \frac{1-(1-\gamma)^{\alpha+1}-\gamma^{\alpha+1}}{\alpha+1} \psi^{\prime \prime}(\gamma u+(1-\gamma) v) d \gamma
\end{aligned}
$$

holds.

This paper is aimed at establishing some new integral inequalities via generalized $s$-convexity on fractal sets. We show that the newly established inequalities are generalized form of Theorem 2 . The new HermiteHadamard type inequalities in the class of functions having derivatives in absolute values are shown to be $s$-convex function on fractal sets. This was achieved using Riemann-Liouville fractional integrals inequalities.

\section{Main results}

Our first main result is obtained by the following theorem.

Theorem 4. Suppose that $\psi:[u, v] \subseteq \mathbb{R}_{+} \rightarrow \mathbb{R}_{+}^{\alpha}$ is a genralized s-convex on $[u, v]$, where $0<s<1$, $u, v \in \mathbb{R}_{+}$and $u \leq v$. If $\psi \in L^{1}([u, v])$, then we obtain

$2^{\alpha s-1} \psi\left(\frac{u+v}{2}\right) \leq \frac{\Gamma(\alpha+1)}{2(v-u)^{\alpha}}\left[J_{u^{+}}^{\alpha} \psi(v)+J_{v^{-}}^{\alpha} \psi(u)\right] \leq\left[\frac{1}{s+1}+\frac{\Gamma(\alpha s+1) \Gamma(\alpha+1)}{\Gamma(\alpha(s+1)+1)}\right] \frac{[\psi(u)+\psi(v)]}{2}$.

Proof. Since $\psi \in G K_{s}^{2}$, we get

$$
\psi\left(\frac{x+y}{2}\right) \leq \frac{\psi(x)+\psi(y)}{2^{\alpha s}}
$$

Substituting $x=\gamma u+(1-\gamma) v$ and $y=(1-\gamma) u+\gamma v$ with $\gamma \in[0,1]$ in inequality (5), we obtain

$$
2^{\alpha s} \psi\left(\frac{x+y}{2}\right) \leq \psi(\gamma u+(1-\gamma) v)+\psi(\gamma v+(1-\gamma) u)
$$

Multiplying both sides of (6) by $\gamma^{\alpha-1}$ and integrating the result inequality with respect to $\gamma$ over $[0,1]$, then we get

$$
\begin{aligned}
\frac{2^{\alpha s}}{\alpha} \psi\left(\frac{x+y}{2}\right) & \leq \int_{0}^{1} \gamma^{\alpha-1} \psi(\gamma u+(1-\gamma) v)+\int_{0}^{1} \gamma^{\alpha-1} \psi(\gamma v+(1-\gamma) u) \\
& =\frac{\Gamma(\alpha)}{(v-u)^{\alpha}}\left[J_{u^{+}}^{\alpha} \psi(v)+J_{v^{-}}^{\alpha} \psi(u)\right] .
\end{aligned}
$$

Then the first inequality in (4) is proved. 
To prove the second inequality in (4), since $\psi \in G K_{s}^{2}$, we get

$$
\psi(\gamma u+(1-\gamma) v) \leq \gamma^{\alpha s} \psi(u)+(1-\gamma)^{\alpha s} \psi(v),
$$

and

$$
\psi(\gamma v+(1-\gamma) u) \leq \gamma^{\alpha s} \psi(v)+(1-\gamma)^{\alpha s} \psi(u) .
$$

Combining the inequalities (8) and (9), we obtain

$$
\begin{aligned}
\psi(\gamma u+(1-\gamma) v)+\psi((1-\gamma) u+\gamma v) & \leq \gamma^{\alpha s} \psi(u)+(1-\gamma)^{\alpha s} \psi(v)+\gamma^{\alpha s} \psi(u)+(1-\gamma)^{\alpha s} \psi(u) \\
& =\left[\gamma^{\alpha s}+(1-\gamma)^{\alpha s}\right][\psi(u)+\psi(v)] .
\end{aligned}
$$

A similar technique used in (6) is applied to inequality (10) to get the following:

$$
\begin{aligned}
\frac{\Gamma(\alpha)}{(v-u)^{\alpha}}\left[J_{u^{+}}^{\alpha} \psi(v)+J_{v^{-}}^{\alpha} \psi(u)\right] & \leq \int_{0}^{1} \gamma^{\alpha-1}\left[\gamma^{\alpha s}+(1-\gamma)^{\alpha s}\right][\psi(u)+\psi(v)] d \gamma \\
& \leq\left[\frac{1}{\alpha(s+1)}+\frac{\Gamma(\alpha s+1) \Gamma(\alpha)}{\Gamma(\alpha(s+1)+1)}\right][\psi(u)+\psi(v),
\end{aligned}
$$

where

$$
\int_{0}^{1} \gamma^{\alpha s+s-1} d \gamma=\frac{1}{\alpha s+\alpha}
$$

and

$$
\int_{0}^{1} \gamma^{\alpha-1}(1-\gamma)^{\alpha s} d \gamma=\frac{\Gamma(\alpha s+1) \Gamma(\alpha)}{\Gamma(\alpha s+\alpha+1)} .
$$

Using inequalities (7) and (11), we proved Theorem 4.

Remark 1. If we set $\kappa=\left[\frac{1}{s+1}+\frac{\Gamma(\alpha s+1) \Gamma(\alpha+1)}{\Gamma(\alpha(s+1)+2)}\right]$ for $0<s \leq 1$, then it is best possible in the second inequality of Theorem 4.

The map $\psi:[0,1] \rightarrow\left[0^{\alpha}, 1^{\alpha}\right]$ given by $\psi(z)=z^{s \alpha}$ is generalization $s$-convex in the second sense,

$$
\frac{\Gamma(\alpha+1)}{2}\left[J_{u^{+}}^{\alpha} \psi(1)+J_{v^{-}}^{\alpha} \psi(0)\right]=\left[\frac{1}{s+1}+\frac{\Gamma(\alpha s+1) \Gamma(\alpha+1)}{\Gamma(\alpha(s+1)+1)}\right],
$$

and

$$
\left[\frac{1}{s+1}+\frac{\Gamma(\alpha s+1) \Gamma(\alpha+1)}{\Gamma(\alpha(s+1)+2)}\right] \frac{[\psi(0)+\psi(1)]}{2}=\left[\frac{1}{s+1}+\frac{\Gamma(\alpha s+1) \Gamma(\alpha+1)}{\Gamma(\alpha(s+1)+1)}\right] .
$$

Corollary 1. Taking $\alpha=1$ in Theorem 4, then the inequality 2 is similar to Theorem 2 introduced by Dragomir and Fitzpatrick [6].

Remark 2. Since

$$
B(u, v)=\frac{\Gamma(u) \Gamma(v)}{\Gamma(u+v)}
$$


Then we have

$$
\begin{aligned}
2^{\alpha s-1} \psi\left(\frac{u+v}{2}\right) \leq \frac{\Gamma(\alpha+1)}{2(v-u)^{\alpha}}\left[J_{u^{+}}^{\alpha} \psi(v)+J_{v^{-}}^{\alpha} \psi(u)\right] & \leq\left[\frac{1}{s+1}+\frac{\Gamma(\alpha s+1) \Gamma(\alpha+1)}{\Gamma(\alpha(s+1)+1)}\right] \frac{[\psi(u)+\psi(v)]}{2} \\
& \leq\left[\frac{1}{s+1}+\beta(\alpha s+1, \alpha+1)\right] \frac{[\psi(u)+\psi(v)]}{2} .
\end{aligned}
$$

Theorem 5. Suppose that $M:[0,1] \rightarrow \mathbb{R}^{\alpha}$ is a mapping given by

$$
M(\gamma)=\frac{\Gamma(\alpha+1)}{(v-u)^{\alpha}}\left[J_{u^{+}}^{\alpha} \psi\left(\gamma v+(1-\gamma) \frac{u+v}{2}\right)+J_{v^{-}}^{\alpha} \psi\left(\gamma u+(1-\gamma) \frac{u+v}{2}\right)\right], \gamma(0,1),
$$

where $\psi:[u, v] \rightarrow \mathbb{R}^{\alpha}$ belongs to $G K_{s}^{2}, s \in(0,1], u, v \in \mathbb{R}_{+}, u<v$ and $\psi \in L^{1}([u, v])$. Then

(i) $M \in G K_{s}^{2}$ on $[0,1]$.

(ii) We have the following inequality:

$$
M(\gamma) \geq 2^{\alpha s} \psi\left(\frac{u+v}{2}\right) .
$$

(iii) We have the following inequality:

$$
M(\gamma) \leq \min \left\{M_{1}(\gamma), M_{2}(\gamma)\right\}, \gamma \in[0,1]
$$

where,

$$
M_{1}(\gamma)=\gamma^{\alpha s} \frac{\Gamma(\alpha+1)}{(v-u)^{\alpha}}\left[J_{u^{+}}^{\alpha} \psi(v)+J_{v^{-}}^{\alpha} \psi(u)\right]+(1-\gamma)^{\alpha s} \psi\left(\frac{u+v}{2}\right),
$$

and

$$
M_{2}(\gamma)=\left[\frac{1}{s+1}+\frac{\Gamma(\alpha s+1) \Gamma(\alpha+1)}{\Gamma(\alpha(s+1)+2)}\right]\left[\psi\left(\gamma u+(1-\gamma) \frac{u+v}{2}\right)+\psi\left(\gamma v+(1-\gamma) \frac{u+v}{2}\right)\right] .
$$

(iv) If $\tilde{M}=\max \left\{M_{1}(\gamma), M_{2}(\gamma)\right\}, \gamma \in[0,1]$, then we have

$$
\tilde{M} \leq\left[\frac{1}{s+1}+\frac{\Gamma(\alpha s+1) \Gamma(\alpha+1)}{\Gamma(\alpha(s+1)+1)}\right]\left[\gamma^{\alpha s}[\psi(u)+\psi(v)]+2^{\alpha s}(1-\gamma)^{\alpha s} \psi\left(\frac{u+v}{2}\right)\right] .
$$

Proof. (i) Let $\gamma_{1}, \gamma_{2} \in[0,1]$ and $\mu_{1}, \mu_{2} \geq 0$ with $\mu_{1}+\mu_{2}=1$, then

$$
\begin{aligned}
M\left(\mu_{1} \gamma_{1}+\mu_{2} \gamma_{2}\right) & =\frac{\Gamma(\alpha+1)}{(v-u)^{\alpha}}\left[J_{u^{+}}^{\alpha} \psi\left(\left(\mu_{1} \gamma_{1}+\mu_{2} \gamma_{2}\right) \frac{u+v}{2}+\left(1-\left(\mu_{1} \gamma_{1}+\mu_{2} \gamma_{2}\right)\right) v\right)\right. \\
& \left.+J_{v^{-}}^{\alpha} \psi\left(\left(\mu_{1} \gamma_{1}+\mu_{2} \gamma_{2}\right) u+\left(1-\left(\mu_{1} \gamma_{1}+\mu_{2} \gamma_{2}\right)\right) \frac{u+v}{2}\right)\right] \\
& \leq \frac{\Gamma(\alpha+1)}{(v-u)^{\alpha}}\left[\mu_{1}^{\alpha s}\left(J_{u^{+}}^{\alpha} \psi\left(\gamma_{1} \frac{u+v}{2}+\left(1-\gamma_{1}\right) v\right)+J_{v^{-}}^{\alpha} \psi\left(\gamma_{1} u+\left(1-\gamma_{1}\right) \frac{u+v}{2}\right)\right)\right. \\
& \left.+\mu_{2}^{\alpha s}\left(J_{u^{+}}^{\alpha} \psi\left(\gamma_{2} \frac{u+v}{2}+\left(1-\gamma_{2}\right) v\right)+J_{v^{-}}^{\alpha} \psi\left(\gamma_{2} u+\left(1-\gamma_{2}\right) \frac{u+v}{2}\right)\right)\right] \\
& =\mu_{1}^{\alpha s} M\left(\gamma_{1}\right)+\mu_{2}^{\alpha s} M\left(\gamma_{1}\right) .
\end{aligned}
$$


(ii) Assume that $\gamma(0,1]$. Then by the change variables $q=\gamma v+(1-\gamma) \frac{u+v}{2}$ and $p=\gamma u+(1-\gamma) \frac{u+v}{2}$, we have

$$
\begin{aligned}
M(\gamma) & =\frac{\Gamma(\alpha+1)}{\gamma^{\alpha}(v-u)^{\alpha}}\left[J_{\left(\gamma v+(1-\gamma) \frac{u+v}{2}\right)^{+}}^{\alpha} \psi\left(\gamma u+(1-\gamma) \frac{u+v}{2}\right)+J_{\left(\gamma u+(1-\gamma) \frac{u+v}{2}\right)^{-}}^{\alpha} \psi\left(\gamma v+(1-\gamma) \frac{u+v}{2}\right)\right] \\
& =\frac{\Gamma(\alpha+1)}{(p-q)^{\alpha}}\left[J_{q^{+}}^{\alpha} \psi(p)+J_{p^{-}}^{\alpha} \psi(q)\right] .
\end{aligned}
$$

Applying the first generalized Hermite-Hadamard inequalities, we obtain

$$
\frac{\Gamma(\alpha+1)}{(p-q)^{\alpha}}\left[J_{q^{+}}^{\alpha} \psi(p)+J_{p^{-}}^{\alpha} \psi(q)\right] \geq 2^{\alpha s} \psi\left(\frac{q+p}{2}\right)=2^{\alpha s} \psi\left(\frac{u+v}{2}\right),
$$

and inequality (12) is obtained.

If $\gamma=0$, the inequality

$$
\psi\left(\frac{u+v}{2}\right) \geq 2^{\alpha s-1} \psi\left(\frac{u+v}{2}\right)
$$

also hold.

(iii) Applying the second generalized Hermite-Hadamard inequalities, we obtain

$$
\begin{aligned}
\frac{\Gamma(\alpha+1)}{(v-u)^{\alpha}}\left[J_{u^{+}}^{\alpha} \psi(v)+J_{v^{-}}^{\alpha} \psi(u)\right] & \leq\left[\frac{1}{s+1}+\frac{\Gamma(\alpha s+1) \Gamma(\alpha+1)}{\Gamma(\alpha(s+1)+1)}\right][\psi(u)+\psi(v)] \\
& =\left[\frac{1}{s+1}+\frac{\Gamma(\alpha s+1) \Gamma(\alpha+1)}{\Gamma(\alpha(s+1)+1)}\right]\left[\psi\left(\gamma u+(1-\gamma) \frac{u+v}{2}\right)\right. \\
& \left.+\psi\left(\gamma v+(1-\gamma) \frac{u+v}{2}\right)\right] \\
& =A_{2}(\gamma), \forall \gamma \in[0,1] .
\end{aligned}
$$

Note that if $\gamma=0$, then the inequality

$$
\psi\left(\frac{u+v}{2}\right)=M(0) \leq M_{2}(0)=2^{\alpha}\left[\frac{1}{s+1}+\frac{\Gamma(\alpha s+1) \Gamma(\alpha+1)}{\Gamma(\alpha(s+1)+1)}\right] \psi\left(\frac{u+v}{2}\right),
$$

holds as it is equivalent to

$$
\left(\left[\frac{\Gamma(\alpha s+\alpha+1)(s+1)}{\Gamma(\alpha s+1) \Gamma(\alpha+1)}\right]-2^{\alpha}\right) \psi\left(\frac{u+v}{2}\right) \leq 0^{\alpha},
$$

and we know that for $s \in(0,1)$.

Since

$$
\psi\left(\gamma u+(1-\gamma) \frac{u+v}{2}\right) \leq \gamma^{\alpha s} \psi(u)+(1-\gamma)^{\alpha s} \psi\left(\frac{u+v}{2}\right)
$$

and

$$
\psi\left(\gamma v+(1-\gamma) \frac{u+v}{2}\right) \leq \gamma^{\alpha s} \psi(v)+(1-\gamma)^{\alpha s} \psi\left(\frac{u+v}{2}\right)
$$


Forall $\gamma \in[0,1]$ and $x \in[u, v]$, then we obtain

$$
\begin{aligned}
M(\gamma) & =\frac{\Gamma(\alpha+1)}{(v-u)^{\alpha}}\left[J_{u^{+}}^{\alpha} \psi\left(\gamma v+(1-\gamma) \frac{u+v}{2}\right)+J_{v^{-}}^{\alpha} \psi\left(\gamma u+(1-\gamma) \frac{u+v}{2}\right)\right] \\
& \leq \gamma^{\alpha s} \frac{\Gamma(\alpha+1)}{(v-u)^{\alpha}}\left[J_{u^{+}}^{\alpha} \psi(v)+J_{v^{-}}^{\alpha} \psi(u)\right]+(1-\gamma)^{\alpha s} \psi\left(\frac{u+v}{2}\right) \\
& =M_{1}(\gamma),
\end{aligned}
$$

and the inequality (13) is proved.

(iv) We have

$$
\begin{aligned}
M_{2}(\gamma) & =\left[\frac{1}{s+1}+\frac{\Gamma(\alpha s+1) \Gamma(\alpha+1)}{\Gamma(\alpha(s+1)+2)}\right]\left[\psi\left(\gamma u+(1-\gamma) \frac{u+v}{2}\right)+\psi\left(\gamma v+(1-\gamma) \frac{u+v}{2}\right)\right] \\
& \leq\left[\frac{1}{s+1}+\frac{\Gamma(\alpha s+1) \Gamma(\alpha+1)}{\Gamma(\alpha(s+1)+1)}\right]\left[\gamma^{\alpha s} \psi(u)+(1-\gamma)^{\alpha s} \psi\left(\frac{u+v}{2}\right)+\gamma^{\alpha s} \psi(v)\right. \\
& \left.+(1-\gamma)^{\alpha s} \psi\left(\frac{u+v}{2}\right)\right] \\
& =\left[\frac{1}{s+1}+\frac{\Gamma(\alpha s+1) \Gamma(\alpha+1)}{\Gamma(\alpha(s+1)+1)}\right]\left[\gamma^{\alpha s}[\psi(u)+\psi(v)]+2^{\alpha s}(1-\gamma)^{\alpha s} \psi\left(\frac{u+v}{2}\right)\right] .
\end{aligned}
$$

Since

$$
\frac{\Gamma(\alpha+1)}{2(v-u)^{\alpha}}\left[J_{u^{+}}^{\alpha} \psi(v)+J_{v^{-}}^{\alpha} \psi(u)\right] \leq\left[\frac{1}{s+1}+\frac{\Gamma(\alpha s+1) \Gamma(\alpha+1)}{\Gamma(\alpha(s+1)+1)}\right] \frac{[\psi(u)+\psi(v)]}{2},
$$

and

$$
(1-\gamma)^{\alpha s} \psi\left(\frac{u+v}{2}\right) \leq 2^{\alpha}(1-\gamma)^{\alpha s}\left[\frac{1}{s+1}+\frac{\Gamma(\alpha s+1) \Gamma(\alpha+1)}{\Gamma(\alpha(s+1)+1)}\right] \psi\left(\frac{u+v}{2}\right)
$$

then

$$
\begin{aligned}
M_{1}(\gamma) & \leq \gamma^{\alpha s}\left[\frac{1}{s+1}+\frac{\Gamma(\alpha s+1) \Gamma(\alpha+1)}{\Gamma(\alpha(s+1)+1)}\right](\psi(u)+\psi(v)) \\
& +2^{\alpha}(1-\gamma)^{\alpha s}\left[\frac{1}{s+1}+\frac{\Gamma(\alpha s+1) \Gamma(\alpha+1)}{\Gamma(\alpha(s+1)+1)}\right] \psi\left(\frac{u+v}{2}\right),
\end{aligned}
$$

and the proof of Theorem 5 is complete.

Corollary 2. Choosing $s=1$ in Theorem 5 , we have

(i)

$$
\begin{aligned}
\frac{\Gamma(\alpha+1)}{(v-u)^{\alpha}}\left[J_{u^{+}}^{\alpha} \psi\left(\gamma v+(1-\gamma) \frac{u+v}{2}\right)\right. & \left.+J_{v^{-}}^{\alpha} \psi\left(\gamma u+(1-\gamma) \frac{u+v}{2}\right)\right] \\
& \leq \min \left\{\gamma^{\alpha} \frac{\Gamma(\alpha+1)}{(v-u)^{\alpha}}\left[J_{u^{+}}^{\alpha} \psi(v)+J_{v^{-}}^{\alpha} \psi(u)\right]\right. \\
& +(1-\gamma)^{\alpha} \psi\left(\frac{u+v}{2}\right),\left[\frac{1}{2}+\frac{(\Gamma(\alpha+1))^{2}}{\Gamma(2(\alpha+1))}\right] \\
& \left.\times\left[\psi\left(\gamma u+(1-\gamma) \frac{u+v}{2}\right)+\psi\left(\gamma v+(1-\gamma) \frac{u+v}{2}\right)\right]\right\} .
\end{aligned}
$$


(ii) Since

$$
\begin{aligned}
& \tilde{M}=\max \left\{\gamma^{\alpha} \frac{\Gamma(\alpha+1)}{(v-u)^{\alpha}}\left[J_{u^{+}}^{\alpha} \psi(v)+J_{v^{-}}^{\alpha} \psi(u)\right]+(1-\gamma)^{\alpha} \psi\left(\frac{u+v}{2}\right),\right. \\
& \left.\left[\frac{1}{2}+\frac{(\Gamma(\alpha+1))^{2}}{\Gamma(2(\alpha+1))}\right]\left[\psi\left(\gamma u+(1-\gamma) \frac{u+v}{2}\right)+\psi\left(\gamma v+(1-\gamma) \frac{u+v}{2}\right)\right]\right\},
\end{aligned}
$$

we get

$$
\tilde{M}=\left[\frac{1}{2}+\frac{\Gamma(\alpha+1) \Gamma(\alpha+1)}{\Gamma(2(\alpha+1))}\right]\left(\gamma^{\alpha}(\psi(u)+\psi(v))+2^{\alpha}(1-\gamma)^{\alpha} \psi\left(\frac{u+v}{2}\right)\right) .
$$

Theorem 6. Let $\psi:[u, v] \subset \mathbb{R}_{+} \rightarrow \mathbb{R}^{\alpha}$ be a differentiable function on $(u, v)$ where $0 \leq u<v$. For some fixed $q \geq 1$, if $\left|\psi^{\prime}\right|^{q}$ is a generalized s-convex on $(u, v)$, we obtain

$$
\begin{aligned}
\left|\frac{\psi(u)+\psi(v)}{2}-\frac{\Gamma(\alpha+1)}{2(v-u)^{\alpha}}\left[J_{u^{+}}^{\alpha} \psi(v)+J_{v^{-}}^{\alpha} \psi(u)\right]\right| & \leq \frac{v-u}{2}\left(\frac{2}{\alpha+1}\right)^{1-\frac{1}{q}} \\
& \times\left(\left[\beta(\alpha+1, \alpha s+1)+\frac{1}{\alpha(s+1)+1}\right]\right. \\
& \left.\times\left[\left|\psi^{\prime}(u)\right|^{q}+\left|\psi^{\prime}(v)\right|^{q}\right]\right)^{\frac{1}{q}} .
\end{aligned}
$$

Proof. Applying Lemma 1, we obtain

$$
\left|\frac{\psi(u)+\psi(v)}{2}-\frac{\Gamma(\alpha+1)}{2(v-u)^{\alpha}}\left[J_{u^{+}}^{\alpha} \psi(v)+J_{v^{-}}^{\alpha} \psi(u)\right]\right|=\frac{v-u}{2}\left|\int_{0}^{1}\left[(1-\gamma)^{\alpha}-\gamma^{\alpha}\right] \psi^{\prime}(\gamma u+(1-\gamma) v) d \gamma\right| .
$$

First, suppose $q=1$. Since the function $\left|\psi^{\prime}(u)\right|$ is a generalized $s$-convex on $(u, v)$, we obtain

$$
\mid \psi^{\prime}\left(\gamma u+(1-\gamma) v \gamma\left|\leq \gamma^{\alpha s}\right| \psi^{\prime}(u)\left|+(1-\gamma)^{\alpha s}\right| \psi^{\prime}(v) \mid .\right.
$$

Therefore,

$$
\begin{aligned}
\left|\int_{0}^{1}\left[(1-\gamma)^{\alpha}-\gamma^{\alpha}\right] \psi^{\prime}(\gamma u+(1-\gamma) v) d \gamma\right| & \leq\left|\psi^{\prime}(u)\right| \int_{0}^{1}\left[(1-\gamma)^{\alpha} \gamma^{\alpha s}+\gamma^{\alpha s+\alpha}\right] d \gamma \\
& +\left|\psi^{\prime}(v)\right| \int_{0}^{1}\left[(1-\gamma)^{\alpha s+\alpha}+\left(1-\gamma^{\alpha s}\right) \gamma^{\alpha}\right] d \gamma \\
& =\left(\beta(\alpha+1, \alpha s+1)+\frac{1}{\alpha s+\alpha+1}\right)\left[\psi^{\prime}(u)+\psi^{\prime}(v)\right] .
\end{aligned}
$$


Suppose that $q>1$, from the power mean inequality

$$
\begin{aligned}
\left|\int_{0}^{1}\left[(1-\gamma)^{\alpha}-\gamma^{\alpha}\right] \psi^{\prime}(\gamma u+(1-\gamma) v) d \gamma\right| & \leq\left|\int_{0}^{1}\left[(1-\gamma)^{\alpha}-\gamma^{\alpha}\right]^{1-\frac{1}{q}}\left[(1-\gamma)^{\alpha}-\gamma^{\alpha}\right]^{\frac{1}{q}} \psi^{\prime}(\gamma u+(1-\gamma) v) d \gamma\right| \\
& \leq\left(\int_{0}^{1}\left|(1-\gamma)^{\alpha}-\gamma^{\alpha}\right| d \gamma\right)^{1-\frac{1}{q}} \\
& \times\left(\int_{0}^{1}\left|(1-\gamma)^{\alpha}-\gamma^{\alpha}\right|\left|\psi^{\prime}(\gamma u+(1-\gamma) v)\right|^{q} d \gamma\right)^{\frac{1}{q}} \\
& \leq\left(\int_{0}^{1}(1-\gamma)^{\alpha}+\gamma^{\alpha} d \gamma\right)^{1-\frac{1}{q}} \\
& \times\left(\int_{0}^{1}\left[(1-\gamma)^{\alpha}+\gamma^{\alpha}\right]\left[\gamma^{\alpha s}\left|\psi^{\prime}(u)\right|^{q}+(1-\gamma)^{\alpha s}\left|\psi^{\prime}(v)\right|^{q}\right] d \gamma\right)^{\frac{1}{q}} \\
& =\left(\frac{2}{\alpha+1}\right)^{1-\frac{1}{q}}\left(\left[\beta(\alpha+1, \alpha s+1)+\frac{1}{\alpha s+\alpha+1}\right]\right. \\
& \left.\times\left[\left|\psi^{\prime}(u)\right|^{q}+\left|\psi^{\prime}(v)\right|^{q}\right]\right)^{\frac{1}{q}} .
\end{aligned}
$$

In view of inequalities (14), (16) and (17) complete the proof of Theorem 6.

Corollary 3. Under the similar conditions of Theorem 6, we get

(i) If $q=s=1$, then

$$
\left|\frac{\psi(u)+\psi(v)}{2}-\frac{\Gamma(\alpha+1)}{2(v-u)^{\alpha}}\left[J_{u^{+}}^{\alpha} \psi(v)+J_{v^{-}}^{\alpha} \psi(u)\right]\right| \leq \frac{v-u}{2}\left[\frac{1}{2 \alpha+1}+\beta(\alpha+1, \alpha+1)\right]\left(\left|\psi^{\prime}(u)\right|+\left|\psi^{\prime}(v)\right|\right) .
$$

(ii) If $q=\alpha=s=1$, then

$$
\left|\frac{\psi(u)+\psi(v)}{2}-\frac{1}{v-u} \int_{u}^{v} \psi(x) d x\right| \leq \frac{v-u}{4}\left(\left|\psi^{\prime}(u)\right|+\left|\psi^{\prime}(v)\right|\right) .
$$

(iii) If $q>1$ and $s=1$

$$
\begin{aligned}
\left|\frac{\psi(u)+\psi(v)}{2}-\frac{\Gamma(\alpha+1)}{2(v-u)^{\alpha}}\left[J_{u^{+}}^{\alpha} \psi(v)+J_{v^{-}}^{\alpha} \psi(u)\right]\right| & \leq \frac{v-u}{2}\left(\frac{2}{\alpha+1}\right)^{1-\frac{1}{q}}\left(\left[\beta(\alpha+1, \alpha s+1)+\frac{1}{\alpha s+\alpha+1}\right]\right. \\
& \left.\times\left[\left|\psi^{\prime}(u)\right|^{q}+\left|\psi^{\prime}(v)\right|^{q}\right]\right)^{\frac{1}{q}} .
\end{aligned}
$$

(iv) If $q>1$ and $\alpha=s=1$

$$
\mid \frac{\psi(u)+\psi(v)}{2}-\frac{1}{v-u} \int_{u}^{v} \psi(x) d x \leq \frac{v-u}{2}\left(\frac{\left|\psi^{\prime}(u)\right|^{q}+\left|\psi^{\prime}(v)\right|^{q}}{2}\right)^{\frac{1}{q}} .
$$


Theorem 7. Let $\psi:[u, v] \subset \mathbb{R}_{+} \rightarrow \mathbb{R}^{\alpha}$ be a differentiable function on $(u, v)$ where $0 \leq u<v$. If $\left|\psi^{\prime}\right|^{q}$ is a generalized $s$-convex on $(u, v)$ for $q>1$, we get

$$
\begin{aligned}
\left|\frac{\psi(u)+\psi(v)}{2}-\frac{\Gamma(\alpha+1)}{2(v-u)^{\alpha}}\left[J_{u^{+}}^{\alpha} \psi(v)+J_{v^{-}}^{\alpha} \psi(u)\right]\right| & \leq \frac{v-u}{2}\left(\frac{2}{\alpha+1}\left[1-\frac{1}{2^{\alpha}}\right]\right)^{1-\frac{1}{q}} \\
& \times\left(\left[\frac{\Gamma(\alpha s+1) \Gamma(\alpha+1)}{\Gamma(\alpha s+\alpha+2)}+\frac{1}{\alpha s+\alpha+1}\right]\right)^{\frac{1}{q}} \\
& \times\left[\left|\psi^{\prime}(u)\right|^{q}+\left|\psi^{\prime}(v)\right|^{q}\right]^{\frac{1}{q}} .
\end{aligned}
$$

Proof. Since $\left|\psi^{\prime}(u)\right|$ is a generalized $s$-convex on $(u, v)$, we obtain

$$
\mid \psi^{\prime}\left(\gamma u+(1-\gamma) v \gamma\left|\leq \gamma^{\alpha s}\right| \psi^{\prime}(u)\left|+(1-\gamma)^{\alpha s}\right| \psi^{\prime}(v) \mid .\right.
$$

From this fact and applying the Hlder's inequality, we have

$$
\begin{aligned}
\left|\int_{0}^{1}\left[(1-\gamma)^{\alpha}-\gamma^{\alpha}\right] \psi^{\prime}(\gamma u+(1-\gamma) v) d \gamma\right| & \leq\left|\int_{0}^{1}\left[(1-\gamma)^{\alpha}-\gamma^{\alpha}\right]^{1-\frac{1}{q}}\left[(1-\gamma)^{\alpha}-\gamma^{\alpha}\right]^{\frac{1}{q}} \psi^{\prime}(\gamma u+(1-\gamma) v) d \gamma\right| \\
& \leq\left(\int_{0}^{\frac{1}{2}}\left[(1-\gamma)^{\alpha}-\gamma^{\alpha}\right] d \gamma+\int_{\frac{1}{2}}^{1}\left[\gamma^{\alpha}-(1-\gamma)^{\alpha}\right] d \gamma\right)^{1-\frac{1}{q}} \\
& \times\left(\int_{0}^{1}\left|(1-\gamma)^{\alpha}-\gamma^{\alpha}\right|\left|\psi^{\prime}(\gamma u+(1-\gamma) v)\right|^{q} d \gamma\right)^{\frac{1}{q}} \\
& \leq\left(\int_{0}^{\frac{1}{2}}\left[(1-\gamma)^{\alpha}-\gamma^{\alpha}\right] d \gamma+\int_{\frac{1}{2}}^{1}\left[\gamma^{\alpha}-(1-\gamma)^{\alpha}\right] d \gamma\right)^{1-\frac{1}{q}} \\
& \times\left(\int_{0}^{1}\left[(1-\gamma)^{\alpha}+\gamma^{\alpha}\right]\left[\gamma^{\alpha s}\left|\psi^{\prime}(u)\right|^{q}+(1-\gamma)^{\alpha s}\left|\psi^{\prime}(v)\right|^{q}\right] d \gamma\right)^{\frac{1}{q}} \\
& =\left(\frac{2}{\alpha+1}\left[1-\frac{1}{2^{\alpha}}\right]\right)^{1-\frac{1}{q}}\left(\left[\frac{\Gamma(\alpha s+1) \Gamma(\alpha+1)}{\Gamma(\alpha s+\alpha+2)}+\frac{1}{\alpha s+\alpha+1}\right]\right)^{\frac{1}{q}} \\
& \times\left[\left|\psi^{\prime}(u)\right|^{q}+\left|\psi^{\prime}(v)\right|^{q}\right]^{\frac{1}{q}} \cdot
\end{aligned}
$$

Thus, the inequalities (14) and (18) complete the proof of Theorem 7.

Theorem 8. Let $\psi:[u, v] \subset \mathbb{R}_{+} \rightarrow \mathbb{R}^{\alpha}$ be a differentiable mapping on $(u, v)$ with $0 \leq u<v$. For some fixed $q>1$, if $\left|\psi^{\prime}\right|^{q}$ is a generalized s-convex on $[u, v]$, then we have:

$\left|\frac{\psi(u)+\psi(v)}{2}-\frac{\Gamma(\alpha+1)}{2(v-u)^{\alpha}}\left[J_{u^{+}}^{\alpha} \psi(v)+J_{v^{+}}^{\alpha} \psi(u)\right]\right| \leq \frac{v-u}{2}\left(\frac{2}{\alpha p+1}\left[1-\frac{1}{2^{\alpha p}}\right]\right)^{\frac{1}{p}}\left(\frac{1}{\alpha s+1}\right)^{\frac{1}{q}}\left(\left|\psi^{\prime}(u)\right|^{q}+\left|\psi^{\prime}(v)\right|^{q}\right)^{\frac{1}{q}}$.

Proof. By applying Hlder's inequality and (15), we obtain 


$$
\begin{aligned}
\left|\int_{0}^{1}\left[(1-\gamma)^{\alpha}-\gamma^{\alpha}\right] \psi^{\prime}(\gamma u+(1-\gamma) v) d \gamma\right| & \leq\left(\int_{0}^{1}\left|(1-\gamma)^{\alpha}-\gamma^{\alpha}\right|^{p} d \gamma\right)^{\frac{1}{p}}\left(\int_{0}^{1}\left|\psi^{\prime}(\gamma u+(1-\gamma) v)\right|^{q} d \gamma\right)^{\frac{1}{q}} \\
& \leq\left(\int_{0}^{\frac{1}{2}}\left[(1-\gamma)^{\alpha}-\gamma^{\alpha}\right]^{p} d \gamma+\int_{\frac{1}{2}}^{1}\left[\gamma^{\alpha}-(1-\gamma)^{\alpha}\right]^{p} d \gamma\right)^{\frac{1}{p}} \\
& \times\left(\int_{0}^{1}\left[\gamma^{\alpha s}\left|\psi^{\prime}(u)\right|^{q}+(1-\gamma)^{\alpha s}\left|\psi^{\prime}(v)\right|^{q}\right] d \gamma\right)^{\frac{1}{q}} \\
& \leq\left(\frac{2}{\alpha p+1}\left[1-\frac{1}{2^{\alpha p}}\right]\right)^{\frac{1}{p}}\left(\frac{\left|\psi^{\prime}(u)\right|^{q}+\left|\psi^{\prime}(v)\right|^{q}}{\alpha s+1}\right)^{\frac{1}{q}} .
\end{aligned}
$$

Finally, from (14) (19) we get the desired result.

Remark 3. From Theorem 8, 6 and 7 we obtain the following inequality for $q>1$

$$
\left|\frac{\psi(u)+\psi(v)}{2}-\frac{\Gamma(\alpha+1)}{2(v-u)^{\alpha}}\left[J_{u^{+}}^{\alpha} \psi(v)+J_{v^{-}}^{\alpha} \psi(u)\right]\right| \leq \min \left\{S_{1}, S_{2}, S_{3}\right\} \frac{(v-u)}{2}\left[\left|\psi^{\prime}(u)\right|^{q}+\left|\psi^{\prime}(v)\right|^{q}\right]^{\frac{1}{q}}
$$

where

$$
\begin{aligned}
& S_{1}=\left(\frac{2}{\alpha+1}\right)^{1-\frac{1}{q}}\left(\left[\beta(\alpha+1, \alpha s+1)+\frac{1}{\alpha(s+1)+1}\right]\right)^{\frac{1}{q}} . \\
& S_{2}=\left(\frac{2}{\alpha+1}\left[1-\frac{1}{2^{\alpha}}\right]\right)^{1-\frac{1}{q}}\left(\left[\frac{\Gamma(\alpha s+1) \Gamma(\alpha+1)}{\Gamma(\alpha(s+1)+2)}+\frac{1}{\alpha(s+1)+1}\right]\right)^{\frac{1}{q}} . \\
& S_{3}=\left(\frac{2}{\alpha p+1}\left[1-\frac{1}{2^{\alpha p}}\right]\right)^{\frac{1}{p}}\left(\frac{1}{\alpha s+1}\right)^{\frac{1}{q}} .
\end{aligned}
$$

Theorem 9. Let $\psi:[u, v] \subset \mathbb{R}_{+} \rightarrow \mathbb{R}^{\alpha}$ be a twice-differentiable function on $(u, v)$ with $0 \leq u<v$. For some fixed $q \geq 1$, if $\left|\psi^{\prime \prime}\right|^{q}$ is a generalized $s$-convex on $(u, v)$, then we get

$$
\begin{aligned}
\left|\frac{\psi(u)+\psi(v)}{2}-\frac{\Gamma(\alpha+1)}{2(v-u)^{\alpha}}\left[J_{u^{+}}^{\alpha} \psi(v)+J_{v^{-}}^{\alpha} \psi(u)\right]\right| & \leq \frac{(v-u)^{2}}{2(\alpha+1)}\left(\frac{\alpha}{\alpha+2}\right)^{1-\frac{1}{q}} \\
& \times\left(\frac{1}{\alpha s+1}-\beta(\alpha s+1, \alpha+2)-\frac{1}{\alpha s+\alpha+2}\right)^{\frac{1}{q}} \\
& \times\left(\left|\psi^{\prime \prime}(u)\right|^{q}+\left|\psi^{\prime \prime}(v)\right|^{q}\right)^{\frac{1}{q}}
\end{aligned}
$$

Proof. Applying Lemma 2, we have

$$
\begin{aligned}
& \left|\frac{\psi(u)+\psi(v)}{2}-\frac{\Gamma(\alpha+1)}{2(v-u)^{\alpha}}\left[J_{u^{+}}^{\alpha} \psi(v)+J_{v^{-}}^{\alpha} \psi(u)\right]\right| \\
= & \frac{(v-u)^{2}}{2} \int_{0}^{1} \frac{1-(1-\gamma)^{\alpha+1}-\gamma^{\alpha+1}}{\alpha+1}\left|\psi^{\prime \prime}(\gamma u+(1-\gamma) v)\right| d \gamma .
\end{aligned}
$$


Firstly, suppose that $q=1$. Since the mapping $\left|\psi^{\prime \prime}\right|$ is generalized $s$-convexity on fractal sets, we obtain

$$
\left|\psi^{\prime \prime}(\gamma u+(1-\gamma) v)\right| \leq \gamma^{\alpha s}\left|\psi^{\prime \prime}(u)\right|+(1-\gamma)^{\alpha s}\left|\psi^{\prime \prime}(v)\right|
$$

Therefore,

$$
\begin{aligned}
& \left|\frac{\psi(u)+\psi(v)}{2}-\frac{\Gamma(\alpha+1)}{2(v-u)^{\alpha}}\left[J_{u^{+}}^{\alpha} \psi(v)+J_{v^{-}}^{\alpha} \psi(u)\right]\right| \\
& \leq \frac{(v-u)^{2}}{2} \int_{0}^{1} \frac{1-(1-\gamma)^{\alpha+1}-\gamma^{\alpha+1}}{\alpha+1}\left(\gamma^{\alpha s}\left|\psi^{\prime \prime}(u)\right|+(1-\gamma)^{\alpha s}\left|\psi^{\prime \prime}(v)\right|\right) d \gamma \\
& =\frac{(v-u)^{2}}{2(\alpha+1)}\left(\frac{1}{\alpha s+1}-\beta(\alpha s+1, \alpha+2)-\frac{1}{\alpha s+\alpha+2}\right)\left(\left|\psi^{\prime \prime}(u)\right|+\left|\psi^{\prime \prime}(v)\right|,\right.
\end{aligned}
$$

where,

$$
\beta(\alpha s+1, \alpha+2)=\beta(\alpha+2, \alpha s+1) .
$$

Secondly, for $q>1$. From Lemma 2 and the power mean inequality, we have

$$
\begin{aligned}
& \left|\frac{\psi(u)+\psi(v)}{2}-\frac{\Gamma(\alpha+1)}{2(v-u)^{\alpha}}\left[J_{u^{+}}^{\alpha} \psi(v)+J_{v^{-}}^{\alpha} \psi(u)\right]\right| \\
\leq & \frac{(v-u)^{2}}{2(\alpha+1)}\left(\int_{0}^{1} 1-(1-\gamma)^{\alpha+1}-\gamma^{\alpha+1}\right)^{1-\frac{1}{q}} \\
\times & \left(\int_{0}^{1} 1-(1-\gamma)^{\alpha+1}-\gamma^{\alpha+1}\left|\psi^{\prime \prime}(\gamma u+(1-\gamma) v)\right|^{q} d \gamma\right)^{\frac{1}{q}} .
\end{aligned}
$$

Hence, from inequalities (21) and (22), we obtain

$$
\begin{aligned}
& \left|\frac{\psi(u)+\psi(v)}{2}-\frac{\Gamma(\alpha+1)}{2(v-u)^{\alpha}}\left[J_{u^{+}}^{\alpha} \psi(v)+J_{v^{-}}^{\alpha} \psi(u)\right]\right| \\
\leq & \frac{(v-u)^{2}}{2(\alpha+1)}\left(\int_{0}^{1} 1-(1-\gamma)^{\alpha+1}-\gamma^{\alpha+1}\right)^{1-\frac{1}{q}}\left(\int_{0}^{1} 1-(1-\gamma)^{\alpha+1}-\gamma^{\alpha+1}\left|\psi^{\prime \prime}(\gamma u+(1-\gamma) v)\right|^{q} d \gamma\right)^{\frac{1}{q}} \\
\leq & \frac{(v-u)^{2}}{2(\alpha+1)}\left(\int_{0}^{1} 1-(1-\gamma)^{\alpha+1}-\gamma^{\alpha+1}\right)^{1-\frac{1}{q}} \\
& \times\left(\int_{0}^{1} 1-(1-\gamma)^{\alpha+1}-\gamma^{\alpha+1}\left[\gamma^{\alpha s}\left|\psi^{\prime \prime}(u)\right|^{q}+(1-\gamma)^{\alpha s}\left|\psi^{\prime \prime}(v)\right|^{q}\right] d \gamma\right)^{\frac{1}{q}} \\
\leq & \frac{(v-u)^{2}}{2(\alpha+1)}\left(\frac{\alpha}{\alpha+2}\right)^{1-\frac{1}{q}}\left(\frac{1}{\alpha s+1}-\beta(\alpha s+1, \alpha+2)-\frac{1}{\alpha s+\alpha+2}\right)^{\frac{1}{q}}\left(\left|\psi^{\prime \prime}(u)\right|^{q}+\left|\psi^{\prime \prime}(v)\right|^{q}\right)^{\frac{1}{q}} .
\end{aligned}
$$

Theorem 10. Let $\psi:[u, v] \subset \mathbb{R}_{+} \rightarrow \mathbb{R}^{\alpha}$ be a twice-differentiable function. If $\left|\psi^{\prime \prime}\right|^{q}$ is a generalized $s$-convex on $(u, v)$, where $0 \leq u<v, s \in(0,1]$ and for some fixed $q>1$, then we get

$$
\begin{aligned}
\left|\frac{\psi(u)+\psi(v)}{2}-\frac{\Gamma(\alpha+1)}{2(v-u)^{\alpha}}\left[J_{u^{+}}^{\alpha} \psi(v)+J_{v^{-}}^{\alpha} \psi(u)\right]\right| & \leq \frac{(v-u)^{2}}{2(\alpha+1)}\left(1-\frac{2}{p(\alpha+1)+}\right)^{\frac{1}{p}} \\
& \times\left(\frac{\left|\psi^{\prime \prime}(u)\right|^{q}+\left|\psi^{\prime \prime}(v)\right|^{q}}{\alpha s+1}\right)^{\frac{1}{q}}
\end{aligned}
$$


where $\frac{1}{p}+\frac{1}{q}=1$.

Proof. From (20), (21) and the Hlders inequality, we have

$$
\begin{aligned}
& \left|\frac{\psi(u)+\psi(v)}{2}-\frac{\Gamma(\alpha+1)}{2(v-u)^{\alpha}}\left[J_{u^{+}}^{\alpha} \psi(v)+J_{b^{-}}^{\alpha} \psi(u)\right]\right| \\
\leq & \frac{(v-u)^{2}}{2(\alpha+1)}\left(\int_{0}^{1}\left(1-(1-\gamma)^{\alpha+1}-\gamma^{\alpha+1}\right)^{p} d \gamma\right)^{\frac{1}{p}}\left(\left|\psi^{\prime \prime}(\gamma u+(1-\gamma) v)\right|^{q} d \gamma\right)^{\frac{1}{q}} \\
\leq & \frac{(v-u)^{2}}{2(\alpha+1)}\left(\int_{0}^{1}\left(1-(1-\gamma)^{p(\alpha+1)}-\gamma^{p(\alpha+1)}\right) d \gamma\right)^{\frac{1}{p}}\left(\left|\psi^{\prime \prime}(u)\right|^{q} \int_{0}^{1} \gamma^{\alpha s} d \gamma+\left|\psi^{\prime \prime}(v)\right|^{q} \int_{0}^{1}(1-\gamma)^{\alpha s} d \gamma\right)^{\frac{1}{q}} \\
\leq & \frac{(v-u)^{2}}{2(\alpha+1)}\left(1-\frac{2}{p(\alpha+1)+}\right)^{\frac{1}{p}}\left(\frac{\left|\psi^{\prime \prime}(u)\right|^{q}+\left|\psi^{\prime \prime}(v)\right|^{q}}{\alpha s+1}\right)^{\frac{1}{q}} .
\end{aligned}
$$

We use

$$
\left(1-(1-\gamma)^{\alpha+1}-\gamma^{\alpha+1}\right)^{q} \leq 1-(1-\gamma)^{q(\alpha+1)}-t^{q(\alpha+1)},
$$

for any $\gamma \in[0,1]$, which follows from

$$
(V-N)^{q} \leq V^{q}-N^{q}
$$

where

$$
V>N \geq 0 \text { and } q \geq 1 .
$$

The proof of Theorem 10 is completed.

The following another Hermit-Hadamard type inequalities of the second derivatives.

Theorem 11. Under the same assumptions of Theorem 10, we have

$$
\begin{aligned}
\left|\frac{\psi(u)+\psi(v)}{2}-\frac{\Gamma(\alpha+1)}{2(v-u)^{\alpha}}\left[J_{u^{+}}^{\alpha} \psi(v)+J_{v^{-}}^{\alpha} \psi(u)\right]\right| & \leq \frac{(v-u)^{2}}{2(\alpha+1)} \\
& \times\left(\frac{1}{\alpha s+1}-\beta(\alpha s+1, q(\alpha+s)+1)-\frac{1}{(\alpha+1) q+\alpha s+1}\right)^{\frac{1}{q}} \\
& \times\left(\left|\psi^{\prime \prime}(u)\right|^{q}+\left|\psi^{\prime \prime}(v)\right|^{q}\right)^{\frac{1}{q}} .
\end{aligned}
$$


Proof. By applying Lemma 2 and the Hlders inequality, we obtain

$$
\begin{aligned}
& \left|\frac{\psi(u)+\psi(v)}{2}-\frac{\Gamma(\alpha+1)}{2(v-b)^{\alpha}}\left[J_{u^{+}}^{\alpha} \psi(v)+J_{v^{-}}^{\alpha} \psi(u)\right]\right| \\
\leq & \frac{(v-u)^{2}}{2(\alpha+1)}\left(\int_{0}^{1} 1 d \gamma\right)^{\frac{1}{p}}\left(\int_{0}^{1}\left(1-(1-\gamma)^{\alpha+1}-\gamma^{\alpha+1}\right)^{q}\left|\psi^{\prime \prime}(\gamma u+(1-\gamma) v)\right|^{q} d \gamma\right)^{\frac{1}{q}} \\
\leq & \frac{(v-u)^{2}}{2(\alpha+1)}\left(\left|\psi^{\prime \prime}(u)\right|^{q} \int_{0}^{1}\left(\gamma^{\alpha s}-(1-\gamma)^{q(\alpha+s)} \gamma^{\alpha s}-\gamma^{q(\alpha+1)+\alpha s}\right) d \gamma\right. \\
& \left.+\left|\psi^{\prime \prime}(v)\right|^{q} \int_{0}^{1}\left((1-\gamma)^{\alpha s}-(1-\gamma)^{q(\alpha+1)+\alpha s}-\gamma^{q(\alpha+1)}(1-\gamma)^{\alpha s}\right) d \gamma\right)^{\frac{1}{q}} \\
\leq & \frac{(v-u)^{2}}{2(\alpha+1)} \times\left(\frac{1}{\alpha s+1}-\beta(\alpha s+1,1+q(\alpha+s))-\frac{1}{(\alpha+1) q+\alpha s+1}\right)^{\frac{1}{q}} \\
\times & \left(\left|\psi^{\prime \prime}(u)\right|^{q}+\left|\psi^{\prime \prime}(v)\right|^{q}\right)^{\frac{1}{q}} .
\end{aligned}
$$

Remark 4. From Theorems 9, 10 and 11, we have

$$
\left|\frac{\psi(u)+\psi(v)}{2}-\frac{\Gamma(\alpha+1)}{2(v-u)^{\alpha}}\left[J_{u^{+}}^{\alpha} \psi(v)+J_{v^{-}}^{\alpha} \psi(u)\right]\right| \leq \min \left\{K_{1}, K_{2}, K_{3}\right\}
$$

where

$$
\begin{aligned}
& K_{1}=\frac{(v-u)^{2}}{2(\alpha+1)}\left(\frac{\alpha}{\alpha+2}\right)^{1-\frac{1}{q}}\left(\frac{1}{\alpha s+1}-\beta(\alpha s+1, \alpha+2)-\frac{1}{\alpha s+\alpha+2}\right)^{\frac{1}{q}}\left(\left|\psi^{\prime \prime}(u)\right|^{q}+\left|\psi^{\prime \prime}(v)\right|^{q}\right)^{\frac{1}{q}} . \\
& K_{2}=\frac{(v-u)^{2}}{2(\alpha+1)}\left(1-\frac{2}{p(\alpha+1)+}\right)^{\frac{1}{p}}\left(\frac{\left|\psi^{\prime \prime}(u)\right|^{q}+\left|\psi^{\prime \prime}(v)\right|^{q}}{\alpha s+1}\right)^{\frac{1}{q}} . \\
& K_{3}=\frac{(v-u)^{2}}{2(\alpha+1)}\left(\frac{1}{\alpha s+1}-\beta(\alpha s+1, q(\alpha+s)+1)-\frac{1}{q(\alpha+1)+\alpha s+1}\right)^{\frac{1}{q}}\left(\left|\psi^{\prime \prime}(u)\right|^{q}+\left|\psi^{\prime \prime}(v)\right|^{q}\right)^{\frac{1}{q}} .
\end{aligned}
$$

\section{Applications to special means}

Using the obtained results, we examine some applications to special means of non-negative numbers $u$ and $v$.

1. The arithmetic mean:

$A=A(u, v)=\frac{u+v}{2} ; u, v \in \mathbb{R}$, with $u, v>0$.

2. The logarithmic mean:

$L(u, v)=\frac{v-u}{\log v-\log u} ; u, v \in \mathbb{R}$, with $u, v>0$.

3. The generalized logarithmic mean:

$$
L_{r}(u, v)=\left[\frac{v^{r+1}-u^{r+1}}{(v-u)(r+1)}\right]^{\frac{1}{r}} ; r \in \mathbb{Z} \backslash\{-1,0\} u, v \in \mathbb{R} \text {, with } u, v>0 .
$$


Using the results obtained in Section 2, and the above applications of means, we get the following proposition.

Proposition 1. Suppose that $r \in \mathbb{Z},|r| \geq 2$ and $u, v \in \mathbb{R}$ such that $0<u<v$, we get the following:

$$
\left|A\left(u^{r}, v^{r}\right)-L_{r}^{r}(u, v)\right| \leq \frac{(v-u)|r|}{2} A\left(|u|^{r-1},|v|^{r-1}\right) .
$$

Proof. This follows from Corollary 3 (ii) wich applied for $\psi(x)=x^{r}$, we get the required result.

Proposition 2. Suppose that $n \in \mathbb{Z},|r| \geq 2$ and $u, v \in \mathbb{R}$ such that $0<u<v$. Then for $q \geq 1$, we get the following:

$$
\left|A\left(u^{r}, v^{r}\right)-L_{r}^{r}(u, v)\right| \leq \frac{(v-u)|r|}{2} A^{\frac{1}{q}}\left(|u|^{q(r-1)},|v|^{q(r-1)}\right) .
$$

Proof. This follows from Corollary 3 (iv) wich applied for $\psi(x)=x^{n}$, we get the required result.

Proposition 3. Suppose that $u, v \in \mathbb{R}$ such that $0<u<v$, then

$$
\left|A\left(u^{-1}, v^{-1}\right)-L(u, v)\right| \leq \frac{(v-u) \mid}{2} A\left(|u|^{-2},|v|^{-2}\right) .
$$

Proof. This follows from Corollary 3 (ii) wich applied for $\psi(x)=\frac{1}{x}$, we obtain the required result.

Proposition 4. Suppose that $u, v \in \mathbb{R}$ such that $0<u<v$, then

$$
\left|A\left(u^{-1}, v^{-1}\right)-L(u, v)\right| \leq \frac{(v-u) \mid}{2} A^{\frac{1}{q}}\left(|u|^{-2 q},|v|^{-2 q}\right) .
$$

Proof. This follows from Corollary 3 (iv) wich applied for $\psi(x)=\frac{1}{x}$, we get the required result.

\section{References}

[1] Yang, X. M. (2001). On E-convex sets, E-convex functions, and E-convex programming. Journal of Optimization Theory and Applications, 109(3), 699-704.

[2] Xie, P., You, K., Tempo, R., Song, S., \& Wu, C. (2018). Distributed convex optimization with inequality constraints over time-varying unbalanced digraphs. IEEE Transactions on Automatic Control, 63(12), 4331-4337.

[3] Almutairi, A., \& Kilı̧̧man, A. (2019). New refinements of the Hadamard inequality on coordinated convex function. Journal of Inequalities and Applications, 2019(1), 192

[4] Hudzik, H., \& Maligranda, L. (1994). Some remarks on s-convex functions. Aequationes Mathematicae, 48(1), 100-111.

[5] Dragomir, S. S., \& Pearce, C. (2003). Selected topics on Hermite-Hadamard inequalities and applications. Mathematics Preprint Archive, 2003(3), 463-817. 
[6] Dragomir, S. S., \& Fitzpatrick, S. (1999). The Hadamard inequalities for s-convex functions in the second sense. Demonstratio Mathematica, 32(4), 687-696.

[7] Agarwal, P., Jleli, M., \& Tomar, M. (2017). Certain Hermite-Hadamard type inequalities via generalized k-fractional integrals. Journal of inequalities and applications, 2017(1), 55.

[8] Sarikaya, M. Z., Set, E., Yaldiz, H., \& Baak, N. (2013). HermiteHadamards inequalities for fractional integrals and related fractional inequalities. Mathematical and Computer Modelling, 57(9-10), 2403-2407.

[9] Mo, H., \& Sui, X. (2014). Generalized-convex functions on fractal sets. In Abstract and Applied Analysis (Vol. 2014). Hindawi.

[10] Kilbas, A. A. A., Srivastava, H. M., \& Trujillo, J. J. (2006). Theory and applications of fractional differential equations (Vol. 204). Elsevier Science Limited.

[11] Chen, F. (2014). On Hermite-Hadamard type inequalities for Riemann-Liouville fractional integrals via two kinds of convexity. Chinese Journal of Mathematics, 2014.

[12] Hudzik, H., \& Maligranda, L. (1994). Some remarks ons-convex functions. Aequationes Mathematicae, 48(1), 100-111. 